\title{
Perfil de tabagismo e frequência de micronúcleos em indivíduos de um município do Sul de Minas Gerais
}

\author{
Smoking profile and frequency of micronuclei in individuals from a municipality of Southern \\ Minas Gerais \\ Perfil de tabaquismo y frecuencia de micronúcleos en individuos de un municipio del sur \\ de Minas Gerais
}

Vanessa Pereira dos Santos ${ }^{1}$, Natan Barbosa Nogueira ${ }^{1}$, José Dias da Silva Neto ${ }^{1}$, Rodrigo Machado Pereira ${ }^{1 *}$.

\section{RESUMO}

Objetivo: Analisar o perfil de tabagismo e a frequência de micronúcleos sobre indivíduos fumantes em comparação aos não-fumantes que residem em um município do sul de Minas Gerais. Métodos: Trata-se de um estudo observacional, envolvendo 60 indivíduos de ambos os gêneros, maiores de 18 anos, divididos igualmente em dois grupos: fumantes e não-fumantes. Foram obtidos dados sobre o perfil de tabagismo mediante a aplicação de questionário. A atividade genotóxica foi avaliada pela análise da frequência de micronúcleos. Células da mucosa oral foram coletadas pelo método citológico convencional, coradas em hematoxilina-eosina ( $\mathrm{HE}$ ), para certificação da qualidade do material, e Giemsa, para análise de micronúcleos. Para análise, foram contabilizadas 1000 células por indivíduo, avaliando-se a frequência de micronúcleos. Os dados foram analisados estatisticamente. Resultados: Mediante a análise, não houve diferença entre os dois grupos para a frequência de micronúcleos $(p=0,839)$. Porém, indivíduos que não consomem álcool fumam mais ( $p=0,045)$, e os que fumam há mais de 30 anos consomem mais cigarros por dia $(p=0,011)$. Conclusão: $O$ perfil de tabagismo da população é heterogêneo, influenciado pelo tempo de uso e o consumo de bebidas alcoólicas, embora pareça não ser um fator exclusivo para o formação de micronúcleos nos indivíduos.

Palavras-chave: Fumar cigarros, Tabaco, Micronúcleo com cromossomo defeituoso, Genotoxicidade.

\begin{abstract}
Objective: Analyze the smoking profile and the frequency of micronuclei on smokers compared to nonsmokers who live in a municipality in the south of Minas Gerais. Methods: This is an observational study, involving 60 individuals of both genders, over 18 years old, divided equally into two groups: smokers and non-smokers. Data on the smoking profile were obtained through the application of a questionnaire. Genotoxic activity was assessed by analyzing the frequency of micronuclei. Cells of the oral mucosa were collected by the conventional cytological method, stained in hematoxylin-eosin (HE), for certification of the quality of the material, and Giemsa, for analysis of micronuclei. For analysis, 1000 cells per individual were counted, evaluating the frequency of micronuclei. Statistical analysis was performed for data analysis. Results: Upon analysis, there was no difference between the two groups for the frequency of micronuclei ( $p$ $=0.839)$. However, it was observed that individuals who do not consume alcohol smoke more $(p=0.045)$, and those who smoke for more than 30 years consume more cigarettes per day $(p=0.011)$. Conclusion: The smoking profile of the population is heterogeneous, influenced by the time of use and the consumption of alcoholic beverages, although it does not seem to be an exclusive factor for the formation of micronuclei in individuals.
\end{abstract}

Key words: Cigarette smoking, Tobacco, Micronuclei, Chromosome-defective, Genotoxicity.

1 Universidade do Vale do Sapucaí (UNIVÁS), Pouso Alegre - MG.

*E-mail: rodrigomachadopereira@yahoo.com.br 


\section{RESUMEN}

Objetivo: Analizar el perfil de tabaquismo y la frecuencia de micronúcleos en fumadores en comparación con no fumadores que viven en un municipio del sur de Minas Gerais. Métodos: Este es un estudio observacional que involucra a 60 individuos de ambos sexos, mayores de 18 años, divididos por igual en dos grupos: fumadores y no fumadores. Los datos sobre el perfil de fumar se obtuvieron mediante la aplicación de un cuestionario. La actividad genotóxica se evaluó analizando la frecuencia de micronúcleos. Las células de la mucosa oral se recogieron por el método citológico convencional, teñido en hematoxilinaeosina (HE), para la certificación de la calidad del material, y Giemsa, para el análisis de micronúcleos. Para el análisis, se contaron 1000 células por individuo, evaluando la frecuencia de micronúcleos. Los datos se analizaron estadísticamente. Resultados: Tras el análisis, no hubo diferencia entre los dos grupos para la frecuencia de micronúcleos ( $p=0,839$ ). Sin embargo, se observó que las personas que no consumen alcohol fuman más $(p=0,045)$, y aquellos que fuman durante más de 30 años consumen más cigarrillos por día $(p=0,011)$. Conclusión: El perfil de tabaquismo de la población es heterogéneo, influenciado por el tiempo de consumo y el consumo de bebidas alcohólicas, aunque no parece ser un factor exclusivo para la formación de micronúcleos en los individuos.

Palabras clave: Fumar cigarrillos, Tabaco, Micronúcleos con defecto cromosómico, Genotoxicidad.

\section{INTRODUÇÃO}

O tabaco é uma das principais causas evitáveis de mortes em todo o mundo, pois leva a óbito até metade de seus usuários. O total estimado é de 8 milhões de pessoas morrem a cada ano, das quais 7 milhões são fumantes diretos e 1,2 milhões são não-fumantes passivos. A grande maioria dos indivíduos que apresentam esse hábito, quase $80 \%$, vivem em países de baixa e média renda. (OPAS/OMS, 2019)

Estima-se que o fumo possa ocasionar cerca de $71 \%$ das mortes por câncer de pulmão, $42 \%$ das doenças respiratórias crônicas e quase $10 \%$ das doenças cardiovasculares (MALTA DC, et al., 2015). Desde a primeira relação estabelecida entre consumo de cigarros e câncer de pulmão no início da década de 1950, os estudos epidemiológicos têm frequentemente identificado novas localizações de câncer com dependências causais ao uso do tabaco. Atualmente, a relação causal do consumo de tabaco equivale a 20 diferentes tipos de tumor, dentre eles: câncer de pulmão, faringe, boca, esôfago e estômago (BITTENCOURT CP, et al., 2017).

A inalação do tabaco expõe o organismo a substâncias tóxicas e agentes oxidativos, que afetam a capacidade antioxidante, protetora das células da mucosa oral dos fumantes (MUNAKATA S, et al., 2018). Os tecidos epiteliais de revestimento podem ser agredidos por agentes químico-físicos, que podem manifestar uma condição de genotoxicidade. Destacam o epitélio oral, que proporciona maior capacidade de absorção, devido às estruturas de suas células (BATISTA CR e CAMPOS JÚNIOR EO, 2014).

Muitos eventos podem induzir processos de carcinogênese, causando alterações genéticas em reguladores dos processos de proliferação celular, diferenciação e apoptose (ALEXANDROV LB, et al., 2016). Dentre os fatores de risco para o desenvolvimento de lesões pré-malignas e malignas na cavidade oral, o fumo tem apresentado uma grande relevância (HASHIBE M, 2020).

Uma das alterações genéticas que ocorrem na cavidade oral de fumantes é a presença de micronúcleos. Tratam-se de corpúsculos constituídos por fragmentos de cromossomos ou cromossomos inteiros, permanecem separados do núcleo principal nas células filhas, ao final do processo de mitose (DE GEUS JL, et al., 2018). A avaliação de micronúcleos proporciona a identificação do eventual aumento na periodicidade de alteração em células que são expostas a uma gama variada de agentes genotóxicos, sobretudo o tabaco (FLORES M e YAMAGUCHI MU, 2008).

A fim de se avaliar as consequências que o hábito de fumar pode causar à saúde humana, é imprescindível que se considere o perfil de tabagismo do espaço amostral a ser avaliado. Atualmente, sabese que diversos fatores estão associados ao consumo e cessação do tabagismo (LEAL T, et al., 2016; MUZI CD, et al., 2018; BILLERBECK NC, BORGES LP, 2019). Consequentemente, estes fatores podem moldar o perfil de distribuição dos fumantes numa população específica (GUIMARÃES ABP, et al., 2017). 
Neste sentido, o presente estudo teve como objetivo avaliar o perfil de tabagismo e identificar ocorrências de modificações celulares induzidas pelo tabaco, por seu efeito genotóxico numa população regional do município de Cambuí no sul de Minas Gerais.

\section{MÉTODOS}

Foram coletados dados e células de 60 indivíduos, sendo 28 do sexo masculino e 32 do sexo feminino, todos residentes em um município selecionado do sul de Minas Gerais. Os participantes foram selecionados em dois grupos, fumantes e não fumantes, de 30 indivíduos cada. Para este estudo, foram incluídos no grupo de fumantes aqueles que relataram fumar diariamente ao menos um cigarro (tabaco) pelo período mínimo de um ano. Ex-fumantes não foram incluídos na amostra.

A abordagem dos participantes foi realizada aleatoriamente em suas residências, praças e locais de trabalho, respeitando todos os princípios éticos estabelecidos pela resolução 466/12 do CONEP. O projeto foi aprovado pelo Comitê de Ética em Pesquisa (23.557.197). Para a realização da pesquisa, a cada participante, foi apresentado o Termo de Consentimento Livre e Esclarecido (TCLE) e o questionário para o levantamento de dados.

Através de entrevista, os participantes foram questionados se fumam ou não. Caso fossem fumantes, buscou-se saber os números aproximado de cigarros por dia e o período de tabagismo em anos, conforme declarado pessoalmente. Também foram perguntados se consumiam bebidas alcoólicas. Idade e sexo também foram registrados.

De cada indivíduo, foram coletadas células da mucosa oral para a confecção de quatro lâminas de material esfoliativo, duas de cada lado (direito e esquerdo) da bochecha, fazendo uso de espátula de madeira estéril. As lâminas, previamente identificadas, foram transferidas para frasco citológico contendo etanol absoluto $99,8 \%$ para fixação.

Para a análise e certificação da qualidade do material obtido, duas lâminas, uma do lado esquerdo e outra do direito, foram coradas em hematoxilina e eosina. Para tanto, o material fixado em álcool foi lavado em banho de água corrente por 1 minuto, seguido de um banho rápido em água destilada. Posteriormente, as lâminas foram coradas com hematoxilina de Harris por 2 minutos e lavadas em água corrente por 5 minutos. As lâminas foram desidratadas com etanol $70 \%$ por 3 minutos e contracoradas por eosina alcoólica por 2 minutos. Por seguinte, o material foi encaminhado para a desidratação, com 1 banho de etanol 95\% durante 5 segundo, e 2 banhos de etanol-isopropanol (1:1) por 30 segundos cada. O material foi clarificado em 3 banhos de xilol por 1 minuto cada. Finalmente, as lâminas foram montadas com lamínula, adicionando-se 1 gota de verniz incolor. A qualidade das amostras foi avaliada por microscopia óptica.

Para a análise de micronúcleos, foi realizada a coloração de Giemsa. As lâminas fixadas foram lavadas em água, dispostas em bandeja, coradas em solução corante Giemsa diluído em água destilada (1:20) por 15 minutos, lavadas com água destilada, secadas e montadas com uma gota de verniz incolor e lamínula.

Foram analisadas 500 células por lâmina ao microscópio óptico, totalizando 1000 células por indivíduo. Foram avaliados campos em sequência na objetiva de 40x. Para serem classificados como micronúcleos, as estruturas observadas apresentaram as seguintes considerações propostas por Barbon FJ, et al. (2015): ser menor que um terço do diâmetro do núcleo principal, estar no mesmo plano, apresentar cor e textura semelhantes ao núcleo, forma ovalada ou arredondada e estar claramente separado do núcleo principal.

Os dados obtidos pela aplicação do questionário e a frequência de micronúcleos foram tabulados no Microsoft Excel 2016 e submetidos à análise estatística. Foram utilizadas medidas de tendência central para variáveis quantitativas e frequência absoluta e relativa para variáveis categóricas. Utilizou-se o programa Minitab (versão 18.1) e Statistical Package for the Social Sciences, Inc. (SPSS, Chicago, USA, versão 22.0). O nível de significância utilizado como critério de aceitação ou rejeição nos testes estatísticos foi de $5 \%$ ( $p<$ 0,05). Para análise dos resultados foram aplicados a análise de correlação de Spearman para estudar a correlação entre os fatores e o teste de Mann-Whitney para avaliar a diferença entre: quantidade de cigarros por tempo de uso, quantidade de cigarros por consumo de álcool e frequência de micronúcleos entre fumante e não fumantes. 


\section{RESULTADOS}

Considerando que a escolha dos participantes tenha sido aleatória, observou-se um padrão específico de distribuição entre os gêneros, faixas etárias e hábito de consumo de bebidas alcoólicas (Figura 1). Ambos os grupos foram constituídos majoritariamente por mulheres $(60 \%$ dos não fumantes e $67 \%$ dos fumantes) com idades entre 21 e 75 anos, das quais aproximadamente a metade declararam consumir álcool ( $58 \%$ das não fumantes e $40 \%$ das fumantes). Os homens entrevistados apresentaram amplitude de faixa etária entre 19 e 73 anos e a maioria consomem bebidas alcoólicas (61\% dos não fumantes e $80 \%$ dos fumantes) (Figura 1A).

A maioria dos tabagistas avaliados neste estudo apresentaram idade entre 41 e 50 anos, ao passo que poucos jovens (18-29 anos) e idosos (>60 anos) fizeram parte deste grupo. Os participantes que não fumam encontram-se mais distribuídos entre as faixas etárias (Figura 1B).

Em um perfil geral, a maioria dos entrevistados declararam consumir álcool. Uma grande maioria dos homens que fumam também consomem bebidas alcoólicas na população analisada, embora menos participantes do grupo dos fumantes de fato afirmaram que bebem (Figura 1C).

Figura 1 - Perfil dos participantes da pesquisa.

\section{A. Sexo dos participantes}

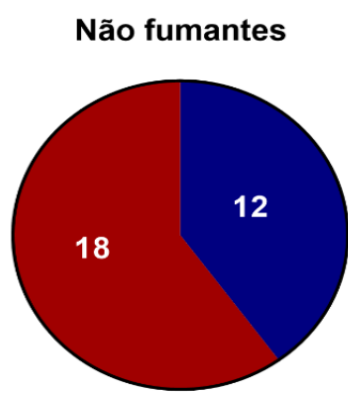

Total $=\mathbf{3 0}$
Fumantes

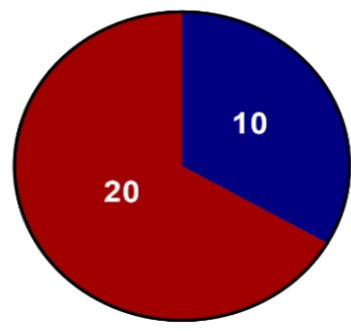

Total $=\mathbf{3 0}$
Homens

Mulheres

B. Idades dos participantes

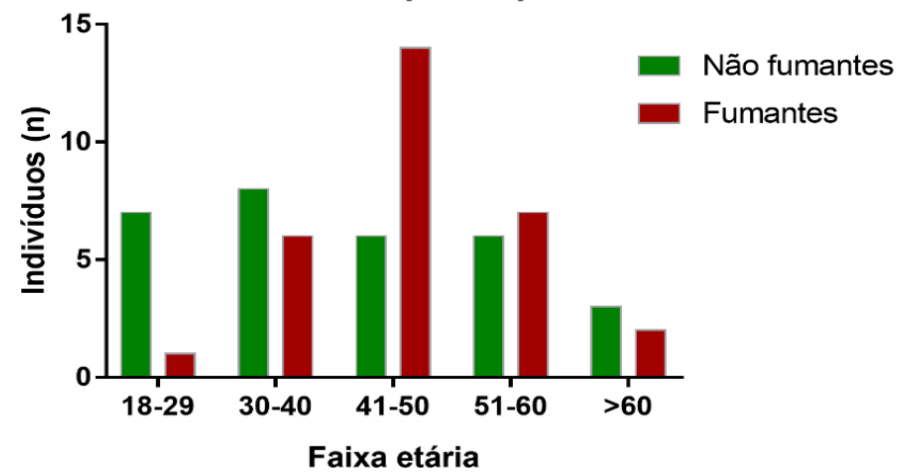

C. Consumidores de bebidas alcoólicas

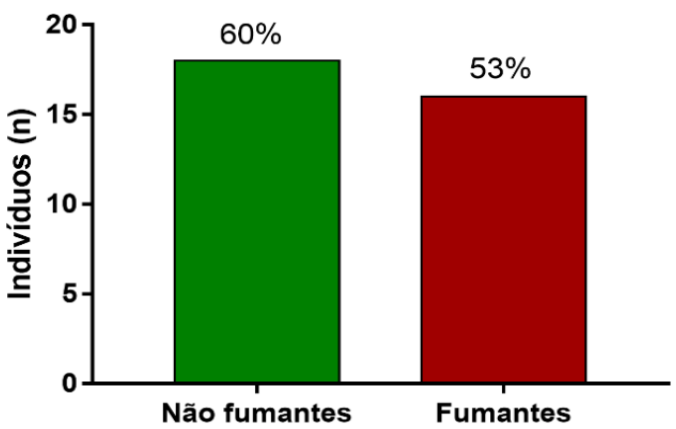

Fonte: Santos VP, et al., 2020. 
A grande maioria dos indivíduos tabagistas entrevistados fumam até 20 cigarros por dia $(n=25)$, e nenhum dos participantes declarou consumir mais que 40 cigarros por dia. Sendo assim, considera-se um perfil majoritário de light smokers (aqueles que fazem uso de poucos cigarros, ao contrário dos heavy smokers) (Figura 2A).

Buscando-se avaliar quantitativamente a associação entre o consumo de cigarros e álcool, a análise estatística apresentou dados significativos entre os fumantes. Foram selecionados dois grupos, fumantes que consomem bebidas alcoólicas $(n=16)$, e fumantes não consumidores de bebidas alcoólicas $(n=14)$. Notou-se uma grande magnitude da mediana do grupo dos indivíduos que não consomem álcool, demonstrando que fumam mais cigarros por dia, tendo uma significância de $p=0,045$. (Figura 2B).

Entre os fumantes pesquisados, há uma grande variação entre o período em que declararam fazer uso do tabaco. As declarações variam entre 3 e 52 anos. Assim como nenhum fumante consome grandes quantidades de cigarro por dia, poucos $(n=3)$ declararam fumar a mais de 40 anos. Embora, deva ser considerado que o período médio de tabagismo do grupo é de 30 anos. (Figura 2C).

A relação quantitativa entre o consumo de cigarros e o tempo de uso mostrou-se foi extremamente relevante. Para tanto, foram analisados em dois grupos: indivíduos que fumam até 30 anos $(n=15)$ e fumantes há mais de 30 anos $(n=15)$. Indivíduos fumantes há mais de 30 anos consomem mais cigarros por dia, já que apresentam mediana o dobro superior ao grupo oposto ( $p=0,011$, Figura 2D).

Figura 2 - Perfil de consumo de cigarros por dia. Valores expressos em mediana (B e C).

\section{A. Consumo geral de cigarros}

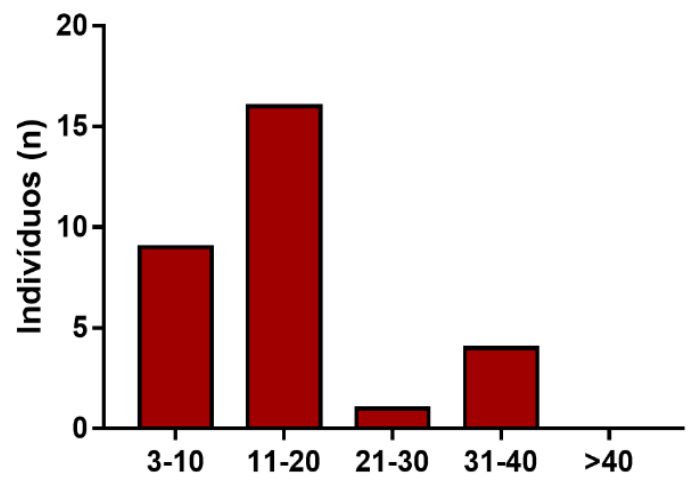

Cigarros por dia ( $n)$

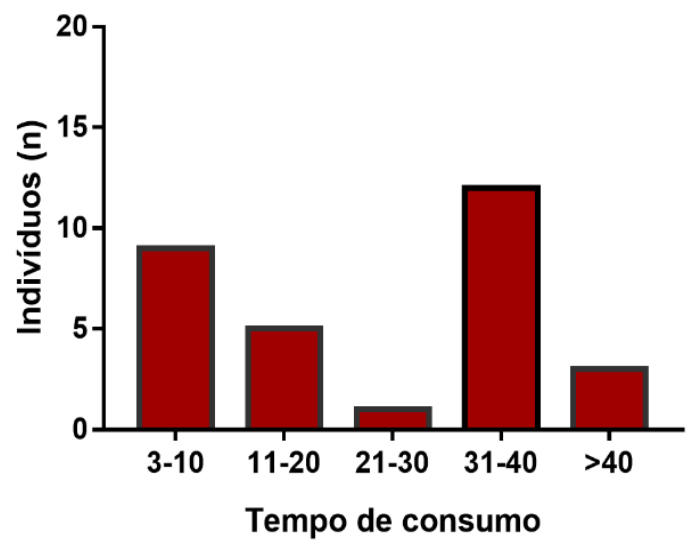

B. Consumo de cigarros x Álcool

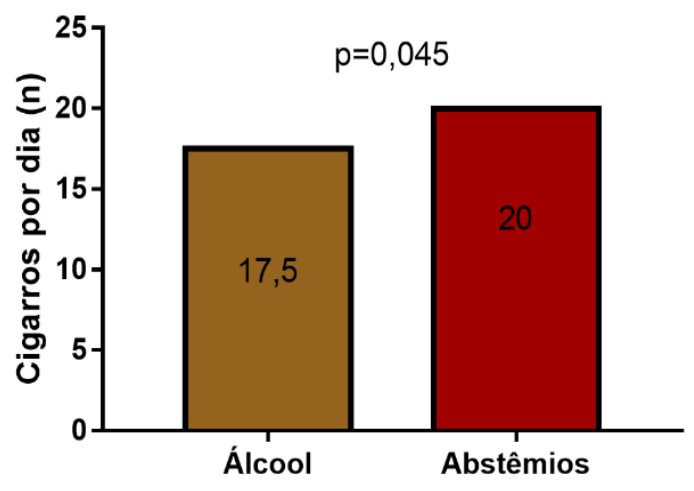

D. Consumo de cigarros $x$ Tempo

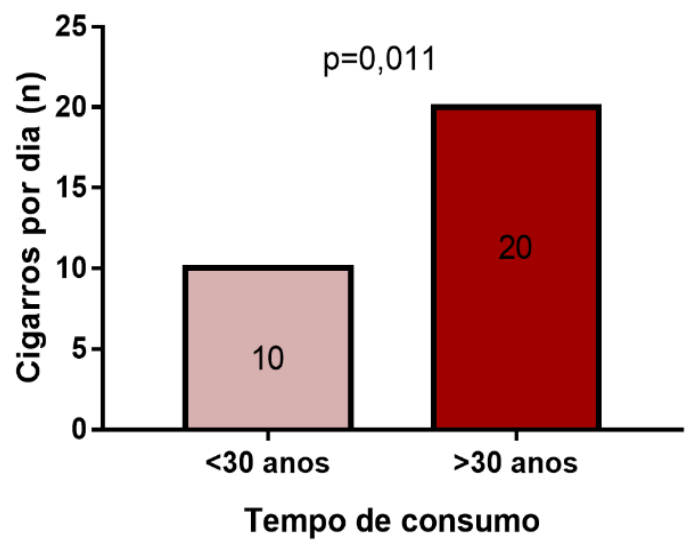

Fonte: Santos VP, et al., 2020. 
Pela análise microscópica das amostras citológicas coletadas e coradas em Giemsa, foi possível identificar micronúcleos em indivíduos de ambos os grupos, fumantes e não fumantes. Embora, nem todos os participantes tiveram micronúcleos identificados pela contagem realizada. Todos os micronúcleos contabilizados apresentaram tamanho inferior a um terço do diâmetro do núcleo principal, cor e textura semelhantes ao núcleo, forma ovalada ou arredondada, separados do núcleo principal, embora pertencessem ao mesmo plano de foco (Figura 3A).

Observou-se numericamente uma pequena superioridade na mediana da frequência de micronúcleos do grupo de fumantes. Em indivíduos fumantes, foi identificado 1,17 micronúcleos entre mil células esfoliadas da mucosa oral. Já os fumantes apresentaram frequência de 1,23\%. Entretanto, após a análise estatística, não foi indicado qualquer significância da frequência de micronúcleos entre fumantes e não fumantes, sendo o valor de $p=0,839$. Não foram apontadas diferenças estatísticas entre os gêneros. Todas as amostras coletadas apresentaram boa qualidade em relação à integridade geral do conteúdo celular, espalhamento das células e ausência de autólise. Estas características foram observadas nas preparações coradas em HE, e indicam ausência de erros e ou artefatos da coleta ou fixação que comprometeriam a análise realizada (Figura 3B).

Figura 3 - Frequência de micronúcleos.

\section{A. Micronúcleo}

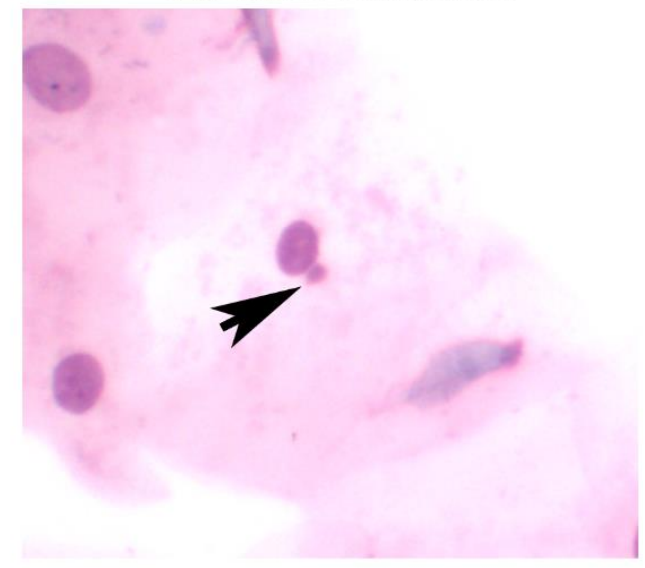

\section{B. Frequência de micronúcleos}

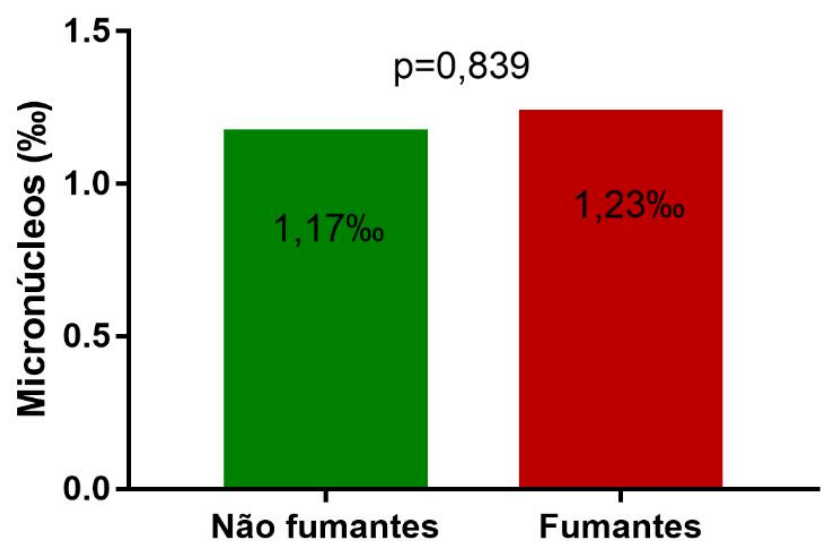

Fonte: Santos VP, et al., 2020.

\section{DISCUSSÃO}

Diversas pesquisas têm sido realizadas no Brasil e no exterior buscando esclarecer o perfil de tabagismo na população (ARRAZOLA RA, et al., 2017; JOSÉ BPS, et al., 2017; MALTA DC, et al., 2019). Os resultados aqui apresentados apontam o perfil de tabagismo de uma população mineira, buscando esclarecer as características dos fumantes e os níveis de consumo de cigarros. Desta maneira, é possível ter uma visão mais abrangente da situação do tabaco considerando também populações de pequenas cidades em processo de desenvolvimento.

De acordo com a Organização Mundial de Saúde, o tabagismo é considerado uma causa importante de morte em todo o mundo (OPAS/OMS, 2019). Esta prática é um fator de risco para doenças cardiovasculares, câncer e doença pulmonar obstrutiva crônica. No Brasil, foi possível observar uma redução geral da mortalidade por estas doenças, que foi associado à redução da exposição dos brasileiros ao cigarro (JOSÉ BPS, et al., 2017). Este resultado pode ser parcialmente decorrente das campanhas antitabagistas que são direcionadas ao público geral, mas principalmente aos mais novos (RAMUSKI CL, 2009).

Acompanhado à redução do consumo de cigarro no país, o número de fumantes juvenis é relativamente inferior aos tabagistas adultos e idosos nas capitais brasileiras (MALTA DC, et al., 2019). Esta distribuição parece ser semelhante à cidade aqui estudada. Porém, há de se destacar que muitos jovens ainda 
apresentam percepção positiva acerca do consumo de cigarros, que reforça a necessidade de políticas públicas (RIBEIRO KCS, et al., 2017).

Quanto ao gênero, estudos apontam que o hábito de tabagismo é mais predominante entre os homens (PAES NL, 2016; MALTA DC, et al., 2019). A superioridade feminina de fumantes no presente estudo é interessante do ponto de vista que houve aumento do número de óbitos causados por câncer relacionado ao tabaco entre mulheres no Brasil (JOSÉ BPS, et al, 2017).

A associação dos hábitos de beber e fumar tem sido estudada recentemente, demonstrando em muitos casos uma predisposição ao consumo de álcool pelos tabagistas (SOUSA BOP, et al., 2018; OLIVEIRA LMFT, et al., 2019). Neste sentido, seria possível concluir que o consumo de cigarros e álcool é diretamente proporcional. Porém, os resultados deste estudo apontam que o tabagismo é inferior nos indivíduos consumidores de bebidas alcoólicas. Esta característica parece ser peculiar da amostra estudada, já que foi demonstrado estatisticamente comparando-se os dois grupos.

Já foi demonstrado que o número de cigarros é relativamente proporcional a dose diária de consumo de bebidas, embora deva ser considerado que esta tendência apresenta variações importantes entre homens e mulheres, negros e brancos. De tal modo, em determinados grupos consumidores de baixas quantidades alcoólicas (1 ou 2 doses diárias), a média de consumo de cigarros chega a ser inferior aos que nunca ou ocasionalmente ingeriram álcool (FRIEDMAN GD, et al., 1991). Embora, altas doses estão sempre relacionadas a um aumento do tabagismo, ao passo que $80 \%$ dos alcoólatras também fumam (ROMBERGER DJ e GRANT K, 2004).

Em outro aspecto, a nicotina, principal substância ativa do tabaco, pode aumentar ou reduzir o consumo próprio de álcool (MCKEE SA e WEINBERGER AH, 2013). Doses crônicas favorecem a dependência alcoólica, enquanto baixas doses de nicotina aplicadas em animais com receptores nicotínicos hipersensibilizados reduziram o consumo voluntário de álcool (HENDRICKSON LM, et al., 2011; LÊ AD, et al., 2003). Da mesma maneira, foi demonstrado experimentalmente em seres humanos que adesivos de nicotina promovem a redução do etilismo em indivíduos alcoólatras tabagistas (MCKEE AS, et al., 2008).

Assim, a relação entre tabagismo e alcoolismo parece ser dose dependente, apresentando comportamento variável. No presente estudo, não foram coletados dados relacionados à quantidade de álcool consumida, embora tenha apresentado uma redução do uso de cigarros entre os consumidores de bebidas alcoólicas. Este resultado deve instigar a investigação mais profunda desta correlação em diferentes condições quantitativas e populações distintas.

Indivíduos que consomem esta quantidade foram considerados como fumantes crônicos (ALVES MGO, et al., 2017; FU S, et al., 2019; LI Z, et al., 2016). O consumo constante e em grande número de cigarros leva a uma das mais comuns e problemáticas formas de dependência em todo mundo. O cigarro é considerado um elemento viciante, tornando-se prazeroso em longo prazo, que por consequência atua sobre o comportamento do indivíduo e promove dificuldades na interrupção (BAUMEISTER RF, 2017).

Este fator está relacionado à dependência criada ao longo do tempo pela nicotina. Por apresentar estrutura química similar à acetilcolina, esta substância age como agonista de alguns receptores colinérgicos (nAChR). A inalação prolongada da fumaça do cigarro mantém a nicotina no organismo, e consequentemente, parte dos receptores nicotínicos são dessensibilizados e tem sua expressão aumentada. Este evento leva ao aumento do desejo fumar e dependência de consumo da nicotina (JILOHA RC, 2019).

Além da nicotina, diversas outras substâncias químicas derivadas do tabaco são absorvidas pelo organismo e promovem efeitos nas células e tecidos. Muitas são considerados genotóxicas, pois promovem quebras nas cadeias de DNA (NAKAYAMA T, et al., 1985). Neste sentido, o teste de micronúcleo tem sido empregado a fim de avaliar os efeitos do tabagismo sobre o material genético, pesquisando a possibilidade de detectar alterações nos indivíduos estudados (BATISTA CR e CAMPOS JÚNIOR EO, 2014).

Os micronúcleos são formandos por quebras cromossômicas ou agentes que afetam o fuso mitótico. Diversos estudos têm demonstrado que a pesquisa destas estruturas em células da mucosa oral pode ser utilizada na detecção de injúrias causadas por agentes ambientais (BENVINDO-SOUZA M, et al., 2017). 
Andrade RB e Campos NA (2020) recentemente apontaram algumas pesquisas que demonstram que a frequência de micronúcleos em indivíduos fumantes é superior aos não-fumantes. Porém, há uma variação significativa nos valores apresentados pelos diferentes estudos. Diversos outros fatores podem influenciar a presença de micronúcleos na cavidade oral, como a deficiência vitamínica (BARBON FJ, et al., 2014; PIYATHILAKE CJ, et al., 1995).

Comparando-se dados da literatura, o número médio de micronúcleos em células esfoliadas da mucosa oral de indivíduos saudáveis sem qualquer tratamento é de 0,3 a 1,72 \%o (NERSESYAN A, 2019), que corresponde aos resultados apresentados.

Um projeto realizado em 30 laboratórios do mundo, baseado em mais de 5000 indivíduos, aponta que o número de micronúcleos pode variar devido à diversos fatores como a idade, exposição a agentes genotóxicos, estado de saúde, dieta, e inclusive o consumo de cigarros. Porém, apenas foi demonstrado uma frequência significativamente superior de micronúcleos em fumantes que consomem mais de 40 cigarros por dia (Frequência relativa $=1,37$ ). (BONASSI S, et al., 2011)

Dos indivíduos fumantes participantes do presente estudo, nenhum declarou fumar mais que quarenta cigarros por dia. Deste modo, é provável que a quantidade de cigarros consumida pela amostra estudada, não foi suficiente para induzir um aumento da frequência de micronúcleos.

\section{CONCLUSÃO}

O presente estudo determinou significância quanto a duas situações evidenciadas na amostra da população. Primeiramente, que o consumo de cigarro é menor em indivíduos que consomem bebida alcoólica. Além disso, o consumo de cigarros é diretamente proporcional ao tempo de tabagismo. $\mathrm{O}$ cigarro compõe-se inúmeras substâncias tóxicas que promovem a instabilidade genética, mas não deve ser levado em consideração como um fator exclusivo para formação de micronúcleos na população estudada. Estes resultados não descartam os problemas resultantes do tabagismo, sendo fator para inúmeras patologias. Neste sentido, seria de grande valia a criação de campanhas e ações antitabagismo, por fim de conscientização da população local sobre os malefícios causados pelo uso do tabaco.

\section{AGRADECIMENTOS}

Nossos mais sinceros agradecimentos à Universidade do Vale do Sapucaí por toda infraestrutura prestada no Laboratório Multidisciplinar de Biologia da Unidade Fátima para realização desta pesquisa. À Gislaine Cristina Scodeler por todo apoio técnico prestado no laboratório.

\section{REFERÊNCIAS}

1. ALEXANDROV LB, et al. Mutational signatures associated with tobacco smoking in human cancer. Science, 2016; 354(6312): 618-622.

2. ALVES MGO, et al. Repair genes expression profile of MLH1, MSH2 and ATM in the normal oral mucosa of chronic smokers. Archives of oral biology, 2017; 73: 60-65.

3. ANDRADE RB, CAMPOS NA. Comparação da frequência de micronúcleos entre indivíduos fumantes e não fumantes: uma revisão sistemática. Jornal Brasileiro de Patologia e Medicina Laboratorial,2020; 56.

4. ARRAZOLA RA, et al. Current tobacco smoking and desire to quit smoking among students aged 13-15 yearsglobal youth tobacco survey, 61 countries, 2012-2015. MMWR. Morbidity and mortality weekly report,2017; 66(20): 533.

5. BARBON FJ, et al. Micronúcleos em fumantes e etilistas. Journal of Oral Investigations,2015; 3(2): 42-45.

6. BATISTA CR, CAMPOS JÚNIOR EO. Avaliação da genotoxicidade em células de pacientes fumantes e não fumantes por meio do teste do micronúcleo. Revista GeTeC, 2014; 3(6).

7. BAUMEISTER RF. Addiction, cigarette smoking, and voluntary control of action: Do cigarette smokers lose their free will?. Addictive behaviors reports, 2017; 5: 67-84.

8. BENVINDO-SOUZA $M$, et al. The micronucleus test for the oral mucosa: global trends and new questions. Environmental Science and Pollution Research, 2017; 24(36): 27724-27730.

9. BILLERBECK NC, BORGES LP. NÍVEL DE ATIVIDADE FÍSICA E TABAGISMO. International Journal of Movement Science and Rehabilitation, 2019; 1(1): 24-32.

10. BITTENCOURT CP, et al. Tabagismo e sua relação com o desenvolvimento de câncer. Revista Científica do ITPAC, Araguaína, 2017; 10(1): 13-18. 
11. BONASSI S, et al. The HUmanMicroNucleus project on eXfoLiated buccal cells (HUMNXL): The role of life-style, host factors, occupational exposures, health status, and assay protocol. Mutation Research/Reviews in Mutation Research, 2011; 728(3): 88-97.

12. DE GEUS JL, et al. Does smoking habit increase the micronuclei frequency in the oral mucosa of adults compared to non-smokers? A systematic review and meta-analysis. Clinical oral investigations, 2018; 22(1): 81-91.

13. GUIMARÃES ABP, et al. A influência da família na iniciação e não utilização de produtos derivados do tabaco: diferenças de gênero. Espaço para Saúde, 2017; 18(2): 70-76.

14. FLORES M, YAMAGUCHI MU. Teste do micronúcleo: uma triagem para avaliação genotóxica. Saúde e Pesquisa, 2009; 1(3): 337-340.

15. FRIEDMAN GD, et al. Alcohol drinking and cigarette smoking: an exploration of the association in middle-aged men and women. Drug and alcohol dependence,1991; 27(3): 283-290.

16. FU S, et al. SF-6D utility scores of smokers and ex-smokers with or without respiratory symptoms attending primary care clinics. Health and Quality of Life Outcomes, 2019; 17(1): 48.

17. HASHIBE M. Risk Factors for Cancer of the Mouth: Tobacco, Betel Quid, and Alcohol. In: Textbook of Oral Cancer. Cham: Springer; 2020; p. 23-30.

18. HENDRICKSON LM, et al. Nicotinic acetylcholine receptors containing the a4 subunit are critical for the nicotineinduced reduction of acute voluntary ethanol consumption. Channels, 2011; 5(2): 124-127.

19. JILOHA RC. Cigarette Smoking: Neurobiology, Addiction and Treatment Implications. Journal of Advanced Research in Psychology \& Psychotherapy (E-ISSN: 2581-5822), 2019; 2(2): 9-16.

20. JOSE BPS, et al. Mortality and disability from tobacco-related diseases in Brazil, 1990 to 2015. RevistaBrasileira de Epidemiologia, 2017; 20: 75-89.

21. LE AD, et al. Nicotine increases alcohol self-administration and reinstates alcohol seeking in rats. Psychopharmacology, 2003; 168(1-2): 216-221.

22. LEAL T, et al. Torches of Freedom: mulheres, cigarros e consumo. Comunicacao, Midia e Consumo, 2016; 13(38): 48.

23. LI Z, et al. Hyper-resting brain entropy within chronic smokers and its moderation by Sex. Scientific reports, 2016; 6 : 29435.

24. MALTA DC, et al. Tendência de fumantes na população Brasileira segundo a Pesquisa Nacional de Amostra de Domicílios 2008 e a Pesquisa Nacional de Saúde 2013. Revista Brasileira de Epidemiologia, 2015; 18: 45-56.

25. MALTA DC, et al. Tendências de indicadores relacionados ao tabagismo nas capitais brasileiras entre os anos de 2006 e 2017. Jornal Brasileiro de Pneumologia, 2019; 45(5).

26. MCKEE SA, et al. Effect of transdermal nicotine replacement on alcohol responses and alcohol selfadministration. Psychopharmacology, 2008; 196(2): 189-200.

27. MCKEE SA, WEINBERGER AH. How can we use our knowledge of alcohol-tobacco interactions to reduce alcohol use?. Annual review of clinical psychology, 2013; 9: 649-674.

28. MUNAKATA S, et al. Oxidative stress responses in human bronchial epithelial cells exposed to cigarette smoke and vapor from tobacco-and nicotine-containing products. Regulatory Toxicology and Pharmacology, 2018; 99: $122-128$.

29. MUZI CD, et al. Gradiente urbano-rural no padrão de consumo e cessação do tabagismo no Brasil. Cadernos de Saúde Pública, 2018; 34: e00077617.

30. NAKAYAMA T, et al. Cigarette smoke induces DNA single-strand breaks in human cells. Nature, 1985; 314(6010): 462-464.

31. NERSESYAN A. Re: Does smoking habit increase the micronuclei frequency in the oral mucosa of adults compared to non-smokers? A systematic review and meta-analysis. de Geus et al., Clin Oral Investig, 2018 Jan; 22 (1): $81-$ 91. Clinical oral investigations, 2019; 23(1): 497-499.

32. OLIVEIRA LMFT, et al. Influência do tabagismo parental no consumo de álcool e drogas ilícitas entre adolescentes. Einstein (São Paulo), $2019 ; 17(1)$.

33. OPAS/OMS. 2019. Folha informativa - Tabaco. Folha informativa atualizada em julho de 2019. Disponível: https://www.paho.org/bra/index.php?option=com_content\&view=article\&id=5641:folha-informativatabaco\&ltemid=1097. Acesso em: 14. Abr. 2020.

34. PAES NL. Fatores econômicos e diferenças de gênero na prevalência do tabagismo em adultos. Ciência\&SaúdeColetiva, 2016; 21: 53-61.

35. PIYATHILAKE CJ, et al. Cigarette smoking, intracellular vitamin deficiency, and occurrence of micronuclei in epithelial cells of the buccal mucosa. Cancer Epidemiology and Prevention Biomarkers, 1995; 4(7): 751-758.

36. RAMUSKI CL. Impactos da campanha antitabagista veiculada em carteiras de cigarro junto aos jovens fumantes. Pensamento\&Realidade, 2009; 24(1).

37. RIBEIRO KCS, et al. Consumo de álcool e tabaco e associação com outras vulnerabilidades em jovens. Psicologia, Saúde \& Doenças, 2017; 18(2): 348-359.

38. ROMBERGER DJ, GRANT K. Alcohol consumption and smoking status: the role of smoking cessation. Biomedicine \& pharmacotherapy, 2004; 58(2): 77-83.

39. SOUSABOP, et al. Associação entre consumo de álcool no padrão binge e tabaco em estudantes de enfermagem. Revista Eletrônica de Enfermagem, 2018; 20. 